\title{
miR-29a inhibits proliferation, invasion, and migration of papillary thyroid cancer by targeting DPP4
}

This article was published in the following Dove Press journal: OncoTargets and Therapy

\section{Yufei Wang* \\ Jie Han* \\ Yuetao Lv \\ Guochao Zhang}

Department of Breast and Thyroid Surgery, Jining NO.I People's Hospital, Affiliated Jining NO.I People's Hospital of Jining Medical University, Jining Medical University, Jining City, Shandong Province 2720II, People's Republic of China

*These authors contributed equally to this work
Correspondence: Guochao Zhang Department of Breast and Thyroid Surgery, Jining NO.I People's Hospital, Affiliated Jining NO.I People's Hospital of Jining Medical University, Jining Medical University, No. 6, Jiankang Road, Jining City, Shandong Province 2720II, People's Republic of China

$\mathrm{Tel} / \mathrm{Fax}+8615865703878$

Email zhangguochao2020@I63.com
Purpose: The purpose of this study was to investigate the effects of miR-29a on papillary thyroid cancer (PTC) and its underlying mechanisms.

Methods: Primary tumor tissues and adjacent tissues of 69 patients with PTC were obtained. Human thyroid cell line Nthy-ori3-1 and PTC cell lines K1, BCPAP, TPC-1 were cultured. K1 cells were transfected and divided into following groups: blank group (without any treatment), miR-29a mimics group, control mimics group, miR-29a inhibitor group, control inhibitor group, DPP4 siRNA group, control siRNA group and miR-29a inhibitor + DPP4 siRNA group. qRTPCR and Western blot were used to detect miR-29a and DPP4 expression. 3-(4,5-dimethylthiazol-2-yl)-2,5-diphenyltetrazolium bromide (MTT) and transwell assay were performed to detect cells proliferation, migration, and invasion. A nude mice xenograft experiment was performed. Results: miR-29a was significantly downregulated in PTC tissues, K1 and TPC-1 cells $(P<0.01)$. DPP4 was significantly upregulated in the miR-29a inhibitor group and significantly downregulated in the miR-29a mimics group $(P<0.01)$. DPP4 was the target gene of miR-29a. miR-29a significantly inhibited K1 cell proliferation, invasion, migration and PTC growth in nude mice by targeting DPP4 $(P<0.01)$.

Conclusion: miR-29a inhibits proliferation, migration, and invasion of PTC by targeting DPP4, which might provide a new target for clinical treatment of PTC.

Keywords: PTC, miR-29a, DPP4, proliferation, migration

\section{Introduction}

Papillary thyroid cancer (PTC) is a common endocrine malignancy that accounts for about $80-85 \%$ of all thyroid cancers. The cure rate of PTC is relatively high compared with other malignant tumors, but the relapse rate after treatment is also very high. Therefore, the recurrence has become the main cause of death in patients with PTC. ${ }^{1}$ Studies showed that the occurrence of PTC was closely related to the expression or mutation of RAS, APAF-1, CD47 and Pax-8/PPAR genes. ${ }^{2}$ miRNAs regulate the expression of most genes and participate in multiple biological processes. miRNAs also undergo abnormal changes in the development of PTC. Previous studies showed that the expressions of miR-574-5p and miR-146b were significantly increased in PTC tissues, which resulted in an increase in the metastatic potential of tumor lymph nodes. Thus, they were used as indicators of PTC preoperative monitoring and postoperative follow-up. ${ }^{3,4}$ In addition, Sondermann et al found that the expression levels of miR-9 and miR-21 in serum and tissues of PTC patients showed significant changes, and these changes were closely associated with tumor size, stage, and metastasis. They also could be used as two indicators of PTC monitoring and postoperative recurrence. ${ }^{5}$ miRNAs regulate cell proliferation, invasion, and metastasis, and also affect apoptosis and 
autophagy in the development and progression of thyroid carcinomas. It has been found that miR-29a played an important regulatory role in many kinds of cancer cells, such as glioma, gastric cancer, and rectal cancer. The mechanism involved cell proliferation and the cell cycle, as well as other physiological processes. ${ }^{6,7} \mathrm{Li}$ et al found that miR29a acted as a tumor suppressor, inhibiting the proliferation and migration of PTC by targeting AKT3 ${ }^{8}$ In recent years, the effect and molecular mechanism of miRNA expression in PTC have received more and more attention.

The dipeptidyl dipeptidase 4 (DPP4), an adenosine deaminase complex protein, was expressed in many organs and cell types. ${ }^{9}$ In addition, it played an important role in various pathophysiologic processes, including diabetes and tumors. Ozog et al found that DPP4 increased significantly in PTC. ${ }^{10}$ However, whether it could be used alone as a biomarker for the diagnosis of papillary thyroid cancer remained to be studied. To explore the mechanism of pathogenesis, we analyzed the expression of miR-29a and its regulation of DPP4 in this study. We hope this research may provide basic information and relevant testing indicators for clinical screening and re-examination.

\section{Material and methods}

\section{Tissues collection and ethical statement}

A total of 69 papillary thyroid carcinoma patients (27 of males and 42 of females) who underwent surgical resection in Jining First People's Hospital from June 2015 to June 2017 were included in this study. The male to female ratio was 1:1.56. Ages ranged from 35 to 56 years, and the average age was 45.5 years. Among them, 46 cases were without lymph node metastases and 23 cases were with lymph node metastases. Primary tumor tissues and adjacent tissues of all these patients were obtained and stored in liquid nitrogen in cryotubes. In this research, patients who had not been treated with thyroidstimulating hormone or iodine 131 before surgery were included, while patients with other serious illnesses, such as other malignancies or systemic infections, were excluded. This study was conducted after obtaining local ethical committee approval of the Affiliated Jining No.1 People's Hospital of Jining Medical University, Jining Medical University. All patients signed informed consent, and this was conducted in accordance with the Declaration of Helsinki.

\section{Cell culture}

Human thyroid cell line Nthy-ori3-1 and human PTC cell lines K1, BCPAP and TPC-1 were purchased from American
Type Culture Collection (American Type Culture Collection (ATCC), Manassas, VA, USA). All cells were cultured in Dulbecco's modified Eagle's medium (DMEM) containing $10 \% \mathrm{FBS}$ in a $\mathrm{CO}_{2}(5 \%)$ incubator at $37^{\circ} \mathrm{C}, 95 \%$ humidity.

\section{Cells transfection}

$\mathrm{K} 1$ cells in logarithmic growth phase were transfected using the lipofectamine 2000 transfection kit (Invitrogen, Thermo Fisher Scientific, Waltham, MA, USA) and the final concentrations of miRNA mimics, miRNA inhibitor and siRNAs were $50 \mathrm{nM}$. Transfection sequences were synthesized by Shanghai Jimao Pharmaceutical Co., Ltd (Shanghai, People's Republic of China). After transfection, cells were grouped as follows: miR-29a mimics group (transfected with miR-29a mimics), control mimics group (transfected with miR-29a negative control mimics), miR-29a inhibitor group (transfected with miR-29a inhibitor), control inhibitor group (transfected with miR-29a negative control inhibitor), DPP4 siRNA group (transfected with DPP4 siRNA), control siRNA group (transfected with DPP4 negative control siRNA), and miR-29a inhibitor + DPP4 siRNA group (cotransfected with miR-29a inhibitor and DPP4 siRNA). K1 cells without any treatment were used as a blank group.

\section{Real-time fluorescence quantitative PCR (qrt-PCR) detection}

The total RNA of cells was extracted using the Trizol kit (Invitrogen). The purity of total RNA was detected using a UV spectrophotometer, and absorbance values at $260 \mathrm{~nm}$ $\left(\mathrm{A}_{260}\right)$ and $280 \mathrm{~nm}\left(\mathrm{~A}_{260}\right)$ were acquired. RNA samples that met $\mathrm{A}_{260} / \mathrm{A}_{280} \geqq 1.70$ standards could be used for subsequent studies. Single-stranded cDNA templates were obtained by reverse transcription using Revert Aid First Strand cDNA Synthesis Kit (Thermo Fisher Scientific) and PCR amplification was then performed. The PCR reaction conditions were as follows: $95^{\circ} \mathrm{C}$ for $60 \mathrm{~s}, 95^{\circ} \mathrm{C}$ for $20 \mathrm{~s}, 58^{\circ} \mathrm{C}$ for $30 \mathrm{~s}, 74^{\circ} \mathrm{C}$ for $30 \mathrm{~s}$. Each experiment was repeated three times. The amplification primer sequences of miR-29a and internal reference U6 snRNA (U6), DPP4 and internal reference GADPH are shown in Table 1.

\section{Western blot detection of DPP4 expression}

K1 cells of the miR-29a mimics group, the control mimics group, the miR-29a inhibitor group, and the control inhibitor group were collected after $48 \mathrm{~h}$ of incubation. Prechilled PBS was used to wash cells three times. Total 
Table I Primer sequences of miR-29a, DPP4, and their internal reference

\begin{tabular}{|l|l|}
\hline $\begin{array}{l}\text { Name of } \\
\text { primer }\end{array}$ & Sequence \\
\hline miR-29a-F & AAAGGATCCGCCATAGAAACCCAGTTTC \\
miR-29a-R & AAAACGCGTCCAAGGGATGAATGTAATTG \\
U6-F & GCTTCGGCAGCACATATACTAAAAT \\
U6-R & CGCTTCACGAATTTGCGTGTCAT \\
DPP4-F & ATTCCAAACAACACACAGT \\
DPP4-R & CTTCATAAACCCAGTCAGT \\
GAPDH-F & GTCGATGGCTAGTCGTAGCATCGAT \\
GAPDH-R & TGCTAGCTGGCATGCCCGATCGATC \\
\hline
\end{tabular}

protein in these cells was extracted by using whole cell lysis buffer (Nanjing KeyGen Biotech Co., Nanjing, China). Subsequently, the protein concentration was determined using a bicinchoninic acid assay (BCA) protein assay reagent kit (Beijing TransGen Biotech Co., Beijing, China). Then, $50 \mu \mathrm{g}$ protein samples were subjected to $10 \%$ SDS-PAGE at $4^{\circ} \mathrm{C}$ for $2 \mathrm{~h}$, followed by transferring to a polyvinylidene difluoride (PVDF) membrane by wet electrotransfer method at $4^{\circ} \mathrm{C}$. The membrane was then blocked with $5 \%$ skim milk-tris-buffered saline-Tween 20 (TBST) for $2 \mathrm{~h}$ at room temperature. Rabbit anti-rat DPP4 primary antibody $(1: 1,000$, Santa Cruz, Germany) was added for $12 \mathrm{~h}$ incubation at $4^{\circ} \mathrm{C}$. After washing with TBST three times, the membrane was incubated with horseradish peroxidase (HRP)-labeled secondary antibody (1:2,000, Vector, USA) for $1 \mathrm{~h}$ at room temperature. GAPDH polyclonal antibody $(1: 1,000$, Proteintech Group, Chicago, IL, USA) served as the control. Finally, blots were developed by a chemiluminescence detection system (ECL-Plus from Amersham, GE Healthcare UK Ltd, Little Chalfont, UK).

\section{Luciferase reporter assay}

Targeted relationships between DPP4 and miR-29a were predicted by TargetScan, and the exact target binding sites were also established. Based on the binding sites, the mutant-type and wild-type sequences of DPP4 3'UTR deletion miR-29a binding sites were designed and cloned. Then, these sequences were ligated into vectors, respectively, to transfect $\mathrm{K} 1$ cells. miR-29a mimics and its negative control mimics were also used to perform transfection on these cells. Based on the differences in transfection sequences, cells were grouped as follows: MT+mimics group (transfected with mutant-type sequences and miR-29a mimics), MT+NC group (transfected with mutant-type sequences and miR-29a negative control mimics), WT+mimics group (transfected with wild-type sequences and miR-29a mimics) and $\mathrm{WT}+\mathrm{NC}$ group (transfected with wild-type sequences and miR-29a negative control mimics). All transfections were performed using lipofectamine 2000. Cells that have been successfully transfected were selected and cultured. Then, they were prepared into cell suspension at a density of $1 \times 10^{5} / \mathrm{mL}$ and seeded in 24 well plates with $1 \mathrm{~mL}$ of suspension per well for $48 \mathrm{~h}$ of incubation at $37^{\circ} \mathrm{C}$. The luciferase activity was determined using a dual luciferase reporter assay system (Promega Corporation, Fitchburg, WI, USA).

\section{MTT assay of cell proliferation}

Cells of each group were cultured in 96-well plates with $1 \times 10^{4}$ cells per well. After being cultured for $24,48,72$, and $96 \mathrm{~h}$ respectively, $20 \mu \mathrm{L}$ 3-(4,5-dimethylthiazol-2-yl)2,5-diphenyltetrazolium bromide (MTT, Sigma-Aldrich Co., St Louis, MO, USA) reaction was added into each well. Then, a total of $150 \mu \mathrm{L}$ DMSO was added into each well after incubation for $4 \mathrm{~h}$. The plates were shaken for $15 \mathrm{~min}$ to fully dissolve the DMSO. $\mathrm{OD}_{570}$ value was obtained by a micro-plate reader under the wavelength of $570 \mathrm{~nm}$.

\section{Transwell detection of cells migration and invasion ability}

After $48 \mathrm{~h}$ of incubation, cells of each group were prepared as serum-free cell suspension and seeded in 24-well plates a density of $2 \times 10^{4}$ cells per well. DMEM containing $10 \%$ FBS was added to the lower chamber of the Transwell chamber. After incubation in a thermostatic incubator for $24 \mathrm{~h}$, the Transwell chamber was removed, and the residual culture medium in the upper chamber was gently removed. Formaldehyde was used to fix cells for 5 min followed by crystal violet staining for $10 \mathrm{~min}$. Five fields were randomly selected for observation and photographing under an inverted microscope. The number of cells that passed through the membrane was considered as the number of migrating cells. For cell invasion assay, Matrigel gel was spread on the upper chamber of the Transwell chamber at first, and the other steps were the same as the migration capability test. The number of cells that passed through the Matrigel gel represented the number of invasive cells.

\section{Nude mice xenograft experiment}

Seventy-two nude mice were purchased from Beijing Chinese Academy of Sciences Laboratory Animal Center and were randomly divided into four groups (blank group, DPP4 
siRNA group, miR-29a mimics group, DPP4 siRNA + miR29 a inhibitor group). All animal assays were processed in accordance with the guide for the care and use of laboratory animals and approved by the ethics committee of Jining First People's Hospital. All these nude mice were housed in sterile laminar flow shelves at $25^{\circ} \mathrm{C}$. Feed and water were sterilized. $\mathrm{K} 1$ cells of the blank group, the DPP4 siRNA group, the miR29a inhibitor group, and the DPP4 siRNA + miR-29a inhibitor group were suspended in PBS at a density of $1 \times 10^{7} / \mathrm{mL}$. Then, these cell suspensions were injected subcutaneously into nude mice with a total volume of $200 \mu \mathrm{L}$. Every week, three nude mice from each group were randomly selected, and subcutaneous tumor mass was removed. Tumor volume was calculated according to the following formula: ${ }^{11,12}$ Volume $\left(\mathrm{mm}^{3}\right)=$ length $(\mathrm{mm}) \times$ width $(\mathrm{mm})^{2} / 2$.

\section{Statistical analysis}

Differences between groups were tested using the Students's $t$-test. SPSS17.0 and GraphPad Prism 5.0 were used for data statistical analysis. $P<0.05$ indicated that the difference between groups was statistically significant.

\section{Results}

\section{miR-29a expression decreased in PTC tissues and cells}

We detected the expression of miR-29a in PTC tumor tissues and adjacent nontumor tissues as well as normal thyroid cells (Nthy-ori3-1) and PTC cell lines (K1, BCPAP, and TPC-1) by qRT-PCR. The results showed that miR-29a expression in tumor tissues was significantly lower than that in adjacent nontumor tissues $(P<0.01)$ (Figure $1 \mathrm{~A})$. We also found that miR-29a relative expression in different

A

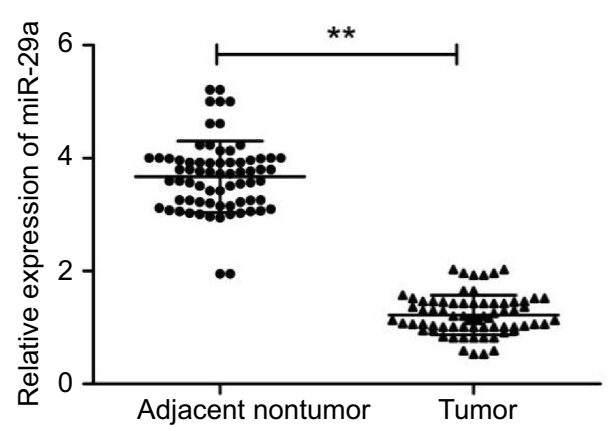

PTC cell lines was lower than that in Nthy-ori3-1. Among them, the relative expression of miR-29a in K1 and TPC-1 cell lines was significantly different from that in normal thyroid cells $(P<0.01)$, and the $\mathrm{K} 1$ cell line had the lowest miR-29a relative expression (Figure 1B).

\section{Cell transfection efficiency}

As shown in Figure 2A, miR-29a expression significantly decreased in the miR-29a inhibitor group compared with the control inhibitor group and the blank group $(P<0.05)$. Similarly, the expression of miR-29a in the miR-29 mimics group was markedly higher than that in the control mimics group and the blank group $(P<0.01)$. When compared with the control inhibitor group, miR-29a expression dramatically increased in the miR-29a inhibitor + DPP4 siRNA group $(P<0.05)$. Figure $2 \mathrm{~B}$ shows that DPP4 mRNA expression in the DPP4 siRNA group was significantly lower than that in the control siRNA group and the blank group $(P<0.01)$. Moreover, DPP4 mRNA expression significantly increased in the miR-29a inhibitor + DPP4 siRNA group compared with the DPP4 siRNA group $(P<0.05)$. All these results confirmed that K1 cells were successfully transfected.

\section{miR-29a inhibited DPP4 expression}

After transfected by miRNA-29a mimics or miR-29a inhibitor, DPP4 relative expression in K1 cells of each group was affected (Figure 3A and B). DPP4 mRNA and protein relative expressions in the miR-29a inhibitor group were significantly higher than those in control inhibitor group $(P<0.01)$. Meanwhile, when compared with the control mimics group, significantly decreased DPP4 mRNA and protein relative expressions were found in the miRNA-29a

B

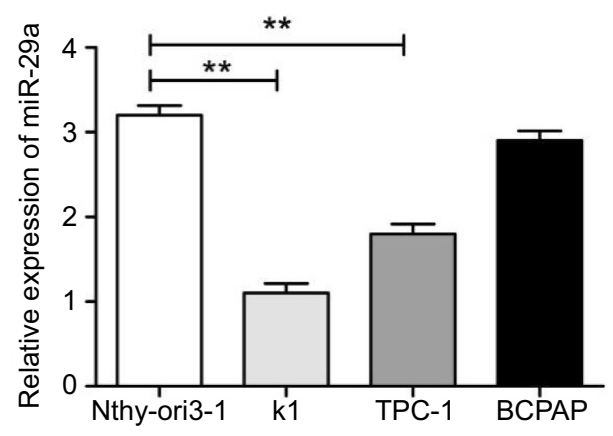

Figure I miR-29a relative expression in PTC tumor tissues, adjacent nontumor tissues, normal thyroid cells (Nthy-ori3-I) and PTC cell lines (KI, BCPAP, and TPC-I) by qRT-PCR. (A) miR-29a relative expression in PTC tumor tissues and adjacent nontumor tissues. (B) miR-29a relative expression in normal thyroid cells (Nthy-ori3-I) and PTC cell lines (KI, BCPAP, and TPC-I). ** P<0.0I.

Abbreviation: PTC, papillary thyroid cancer. 
A

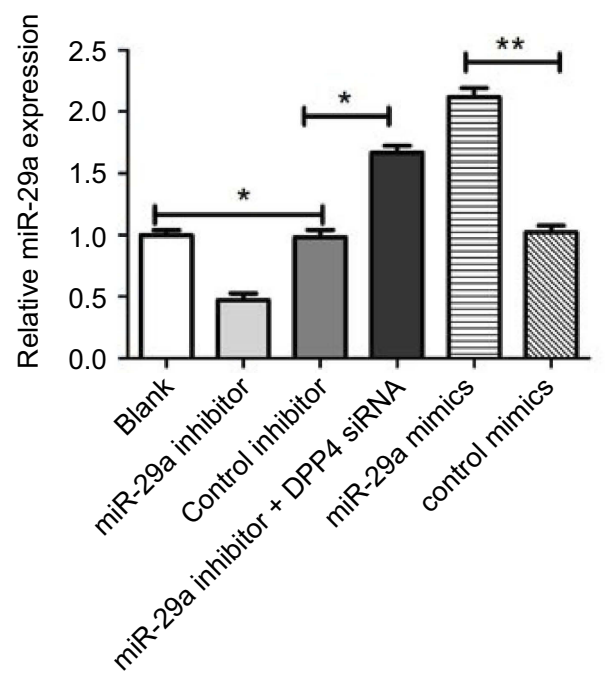

B

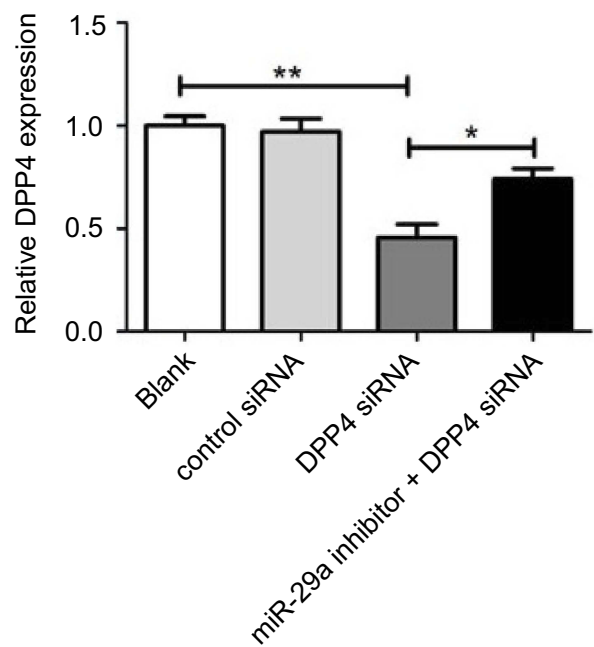

Figure 2 The miR-29a and DPP4 expression in KI cells after transfection. (A) The expression of miR-29a in KI cells. (B) The expression of DPP4 in KI cells. $* P<0.05$ or $* * P<0.01$.

A

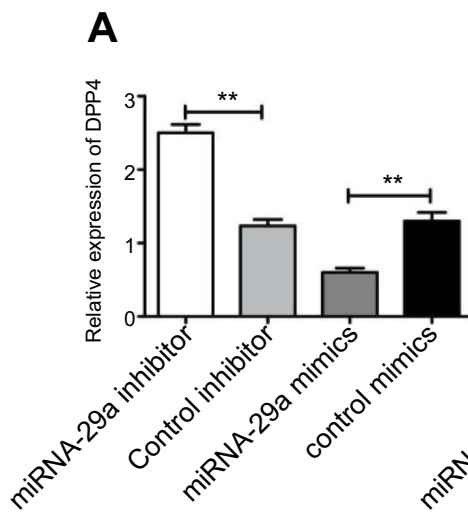

B

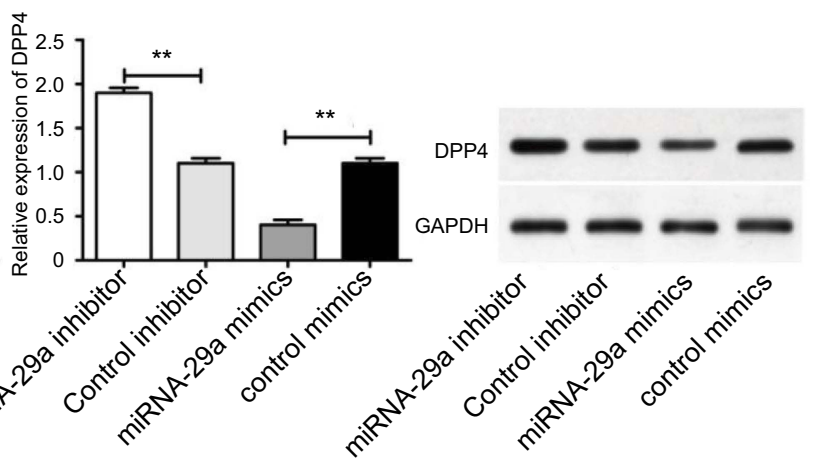

Figure 3 Effect of miR-29a on DPP4 expression (A) DPP4 mRNA expression was tested by qRT-PCR. (B) DPP4 protein expression was measured by Western blot. **P<0.0I.

mimics group $(P<0.01)$. The results indicated that miR-29a could effectively reduce the expression of DPP4 in K1 cells.

\section{DPP4 was the target gene of miR-29a}

TargetScan predicted that the binding site of DPP4 mRNA and miR-29a was the $3^{6}$-UTR region (Figure 4A). According to the results of the luciferase reporter assay, we found that the difference of luciferase activity between the $\mathrm{MT}+$ mimics group, and the $\mathrm{MT}+\mathrm{NC}$ group was not statistically significant, while significantly lower luciferase activity in the WT + mimics group was observed when compared with that in the WT $+\mathrm{NC}$ group $(P<0.01)$ (Figure 4B). Based on these results, we could confirm the regulatory effect of miR-29a on DPP4 expression.

\section{miR-29a inhibited $\mathrm{KI}$ cells proliferation by targeting DPP4}

MTT assay results showed that $\mathrm{OD}_{570}$ values of each group were all increased over time. It also should be noted that $\mathrm{OD}_{570}$ values of the miR-29a inhibitor group were higher than those of the blank group with statistical differences $(P<0.05)$ at $48 \mathrm{~h}$ and $72 \mathrm{~h}$. When compared with the blank group, a significantly higher $\mathrm{OD}_{570}$ value in the miR-29a inhibitor group $(P<0.01)$ was found at $96 \mathrm{~h}$. However, the $\mathrm{OD}_{570}$ value of the DPP4 siRNA group was obviously lower than that of the blank group $(P<0.05)$ at the same time. The difference of $\mathrm{OD}_{570}$ values between the miR-29a inhibitor + DPP4 siRNA group and the blank group was not statistically significant all the time (Figure 5). 
A

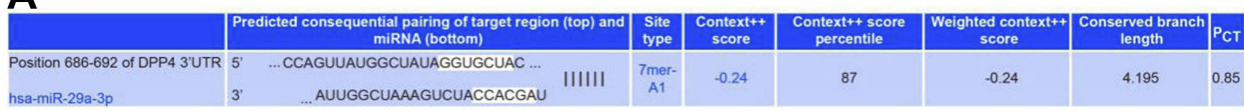

B

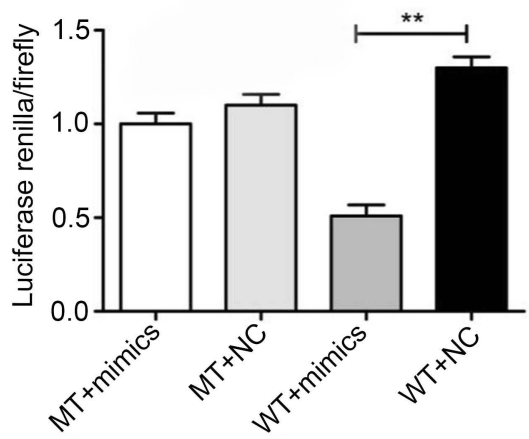

Figure 4 miR-29a was directly targeted on DPP4. (A) Prediction of binding sites for DPP4 and miR-29a by TargetScan. (B) Luciferase reporter assay to confirm the regulatory effect of miR-29a on DPP4 expression. $* * P<0.01$.

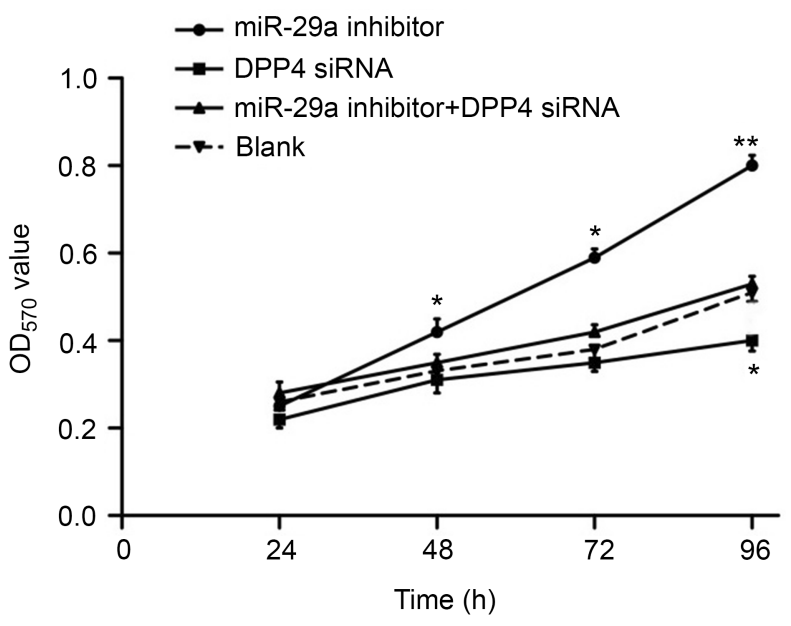

Figure 5 miR-29a inhibited $\mathrm{KI}$ cells proliferation by targeting DPP4. $* P<0.05$ or $* * P<0.0$ I when compared with the blank group.

\section{miR-29a inhibited $\mathrm{KI}$ cells invasion and migration by targeting DPP4}

According to the Transwell experiment, we observed that the cell count of migration and invasion in the miR-29a inhibitor group was both significantly higher than that in the blank group $(P<0.01)$. In addition, dramatically lower numbers of migratory and invasive cells in the DPP4 siRNA group were found when compared with that in the blank group $(P<0.05)$. However, there was no statistical difference in the number of migratory and invasive cells between the miR-29a inhibitor + DPP4 siRNA group and the blank group (Figure 6A and B).

\section{miR-29a inhibited PTC growth in nude mice by targeting DPP4}

In this study, K1 cells of the blank group, the miR-29a inhibitor group, the DPP4 siRNA group, and the miR-29a inhibitor + DPP4 siRNA group were transplanted into nude mice by subcutaneous injection. The results showed that, from 4 weeks after transplantation, the tumor volume in the miR-29a inhibitor group was always larger than that in the blank group $(P<0.05)$. Furthermore, in the sixth week, significantly lower tumor volume in the DPP4 siRNA group was found when compared with that of the blank group $(P<0.01)$. From 1 to 6 weeks, statistically significant tumor volume differences were not observed between the miR-29a inhibitor + DPP4 siRNA group and the blank group (Figure 7A and B).

\section{Discussion}

PTC is a malignant tumor with a high recurrence rate, which causes a great risk to human health and life. ${ }^{13}$ Its thorough treatment at the molecular level has caused clinical concern. However, the discovery of the exact therapeutic target remains a difficult problem in clinical research. In this paper, we found that miR-29a was a new therapeutic target for PTC, which could exert a suppressive effect on PTC cell proliferation, migration, and invasion. The underlying mechanism was through inhibiting the expression of DPP4.

miRNAs play an important role in cell differentiation, proliferation, and apoptosis. Meanwhile, it is significantly associated with various clinical diseases such as cardiovascular, nervous system and metabolic diseases. Abnormal expression of specific miRNA may lead to the occurrence and development of tumors. Studies have shown that some miRNAs such as miR-221, miR-222, miR-146b, and miR199a were abnormally expressed in PTC. miR-29a participated in multiple biological processes, and has been 
A
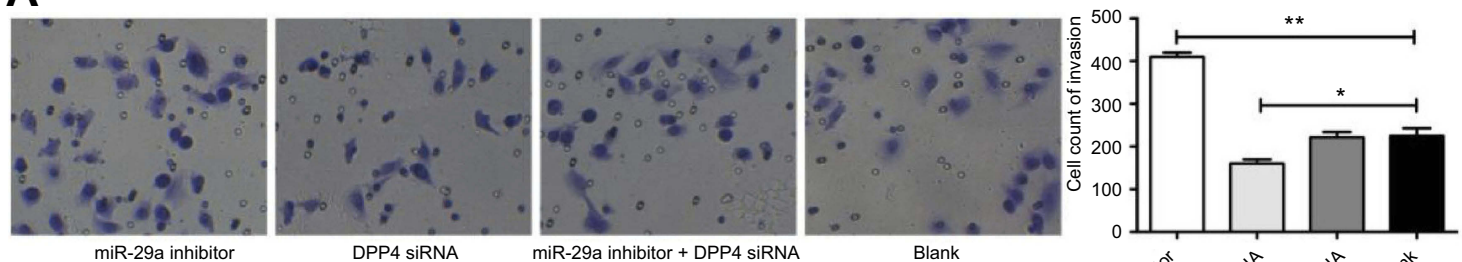

B
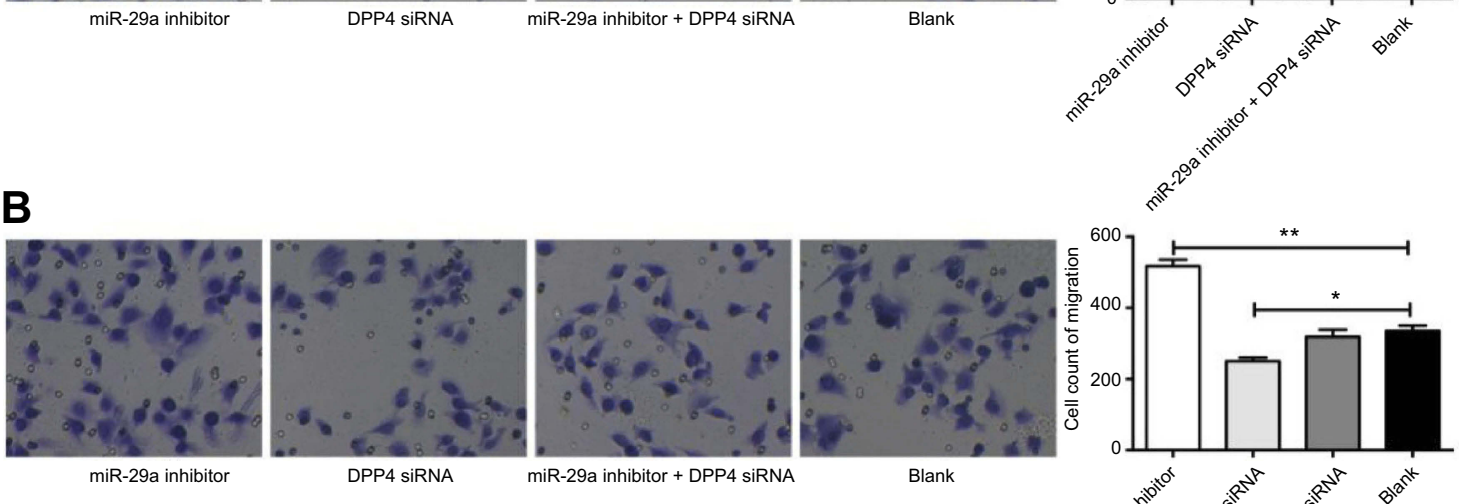

miR-29a inhibitor

DPP4 siRNA

miR-29a inhibitor + DPP4 siRNA

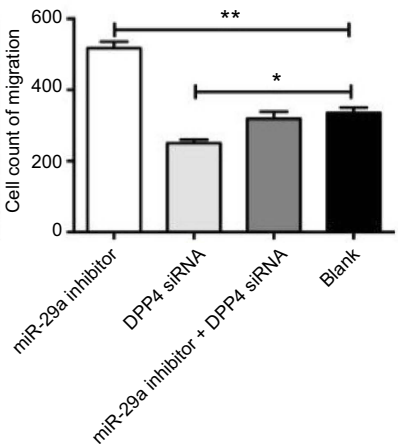

Figure 6 miR-29a inhibited KI cells invasion and migration by targeting DPP4. (A) Detection of KI cells invasion by the transwell experiment. (B) Detection of KI cells migration by the transwell experiment. $* P<0.05$ or $* * P<0.01$ when compared with the blank group.

A

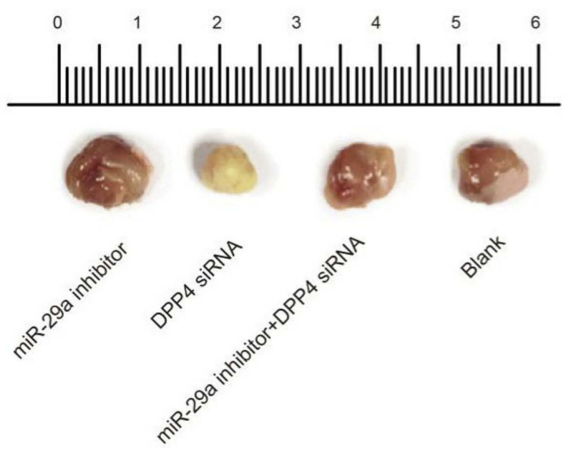

B

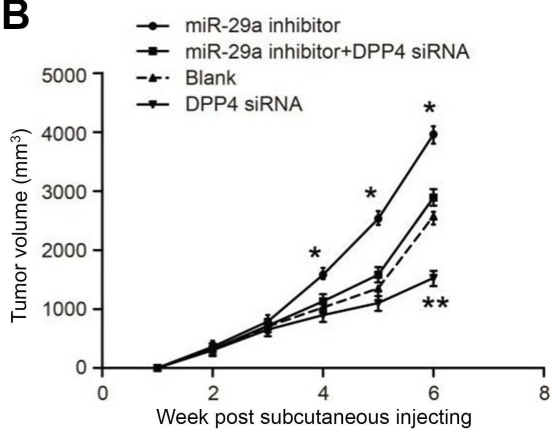

Figure 7 miR-29a inhibited PTC growth in nude mice by targeting DPP4. (A) Tumor tissues in nude mice of each group 6 weeks after transplantation. (B) Tumor volume in nude mice of each group at different time after transplantation. $* P<0.05$ or $* * P<0.01$ when compared with the blank group.

Abbreviation: PTC, papillary thyroid cancer.

implicated in various biological processes in colon, prostate, and gastric cancers, including cell proliferation, cell cycle, as well as cell migration and invasion. ${ }^{6,14,15}$ Our results revealed that in PTC tumor tissues, miR-29a expression was significantly lower than in adjacent nontumor tissues. In different PTC cell lines, the relative expression of miR29a was lower than that in normal thyroid cells. Among them, miR-29a relative expression in $\mathrm{K} 1$ and TPC-1 cell lines was significantly lower than that in normal thyroid cells. $\mathrm{Li}$ et al also found that miR-29a acted as a tumor suppressor for PTC by targeting the AKT3 gene. ${ }^{16}$ In hepatoma cells, miR-29a-3p could inhibit cell proliferation and migration by downregulating IGF1R, and similarly, miR29a could inhibit cell proliferation and induce apoptosis by inhibiting STAT3 expression in rheumatoid arthritis. ${ }^{17}$ Moreover, miR-29a-3p could inhibit proliferation and invasion of papillary thyroid carcinoma by suppressing NF- $\mathrm{B}$ signaling via direct targeting of OTUB2. ${ }^{18}$ Ma et al found in 
their research that miR-29a could target schwannoma cells, thereby inhibiting cell proliferation and migration. ${ }^{19}$ In this paper, we observed that miR-29a could inhibit K1 cells proliferation, migration, invasion and tumor growth in nude mice by inhibiting the expression of DDP4.

DPP4 is adenosine deaminase complex protein. Recent studies have found that DPP4 played an important role in various pathophysiologic processes, such as the development of inflammatory diseases, diabetes, and even cancers. $^{20}$ Researchers havealsofound that DPP4 was closely related to immune regulation, signal transduction, and apoptosis. $^{21}$ There was an article revealing that DPP4 inhibitors had a therapeutic effect on type 2 diabetes, which was an incretin-based drug. Currently, several DPP4 inhibitors have been applied clinically, and they could stimulate pancreatic b cells to secrete insulin by increasing incretin hormones level, such as glucagon-like peptide. $^{22}$ DPP4 was also reported to have a renal protective effect, and for diabetic nephropathy, DPP4 inhibitors could reduce proteinuria and glomerulosclerosis. ${ }^{23-25}$ In a mouse model of pancreatic beta cell injury, DPP4 inhibitors could play a protective role on the cells and promote the proliferation of islet beta cells. ${ }^{26}$ Wronkowitz experimentally confirmed that DPP4 could enhance the proliferation and immunity of human smooth muscle cells through protease-activated receptors. ${ }^{27}$ Davies et al also reported that DPP4 had biological effects on tumor cells, such as malignant mesothelioma, colorectal cancer, as well as chronic myeloid leukemia, which was associated with several tumors malignant activity. ${ }^{28}$ However, few studies have confirmed the role of DPP4 in PTC currently. In our research, we concluded that miR-29a could inhibit the proliferation, invasion, and migration of PTC cells as well as tumor growth in nude mice by inhibiting the expression of DPP4.

\section{Conclusion}

In summary, miR-29a can inhibit the proliferation, invasion, and migration of PTC cells as well as tumor growth in nude mice by targeting DPP4. This study provides a new target for the clinical control of proliferation, migration, and invasion of PTC at the gene level. The development of PTC is a complex process involving various mechanisms. We only investigated the relationship between miR-29a and DDP4 in the study. In the future, we will study more targets, such as NF- $\mathrm{B}$ and OTUB2.

\section{Ethics approval and consent to participate}

This study was conducted after obtaining local ethical committee approval of the Affiliated Jining No.1 People's Hospital of Jining Medical University, Jining Medical University. All patients signed informed consent, and this was conducted in accordance with the Declaration of Helsinki.

\section{Acknowledgment}

There is no funding in the research.

\section{Author contributions}

All authors contributed to data analysis, drafting and revising the article, gave final approval of the version to be published, and agree to be accountable for all aspects of the work.

\section{Disclosure}

The authors report no conflicts of interest in this work.

\section{References}

1. Xing M, Alzahrani AS, Carson KA, et al. Association between BRAF V600E mutation and recurrence of papillary thyroid cancer. J Clin Oncol. 2015;33:42-50. doi:10.1200/JCO.2014.56.8253

2. Raman P. Koenig RJ Pax-8-PPAR- $\gamma 3$ fusion protein in thyroid carcinoma. Nat Rev Endocrinol. 2014;10. doi:10.1038/nrendo.2014.115

3. Wang X, Lu X, Geng Z, et al. LncRNA PTCSC3/miR-574-5p governs cell proliferation and migration of papillary thyroid carcinoma via Wnt/ $\beta$-catenin signaling. J Cell Biochem. 2017. doi:10.1002/ jcb. 26142

4. Chou CK, Yang KD, Chou FF, et al. Prognostic implications of miR-146b expression and its functional role in papillary thyroid carcinoma. J Clin Endocrinol Metab. 2013;98:196-205. doi:10.1210/ jc.2012-2666

5. Sondermann A, Andreghetto FM, Moulatlet ACB, et al. MiR-9 and miR-21 as prognostic biomarkers for recurrence in papillary thyroid cancer. Clin Exp Metastasis. 2015;32:521. doi:10.1007/s10585-0159724-3

6. Yun C, Su WY, Jing X, et al. MiR-29a inhibits cell proliferation and induces cell cycle arrest through the downregulation of p42.3 in human gastric cancer. PLoS One. 2011;6:e25872. doi:10.1371/journal. pone. 0025872

7. Zhao D, Jiang $\mathrm{X}$, Yao C, et al. Heat shock protein 47 regulated by miR-29a to enhance glioma tumor growth and invasion. $J$ Neurooncol. 2014;118:39-47. doi:10.1007/s11060-014-1412-7

8. Feng W, Ni H, Feng S, et al. Overexpression of lncRNA AFAP1-AS1 correlates with poor prognosis and promotes tumorigenesis in colorectal cancer. Biomed Pharmacother. 2016;81:152-159. doi:10.1016/j. biopha.2016.04.009

9. Noels H, Theelen W, Sternkopf M, et al. Reduced post-operative DPP4 activity associated with worse patient outcome after cardiac surgery. Sci Rep. 2018;8:11820. doi:10.1038/s41598-018-30235-w

10. Ozóg J, Jarzab M, Pawlaczek A, et al. Expression of DPP4 gene in papillary thyroid carcinoma. Endokrynol Pol. 2006;57(Suppl A):12. 
11. Ma S, Jia W. Ni S miR-199a-5p inhibits the progression of papillary thyroid carcinoma by targeting SNAI1. Biochem Biophys Res Commun. 2018;497:S0006291X18302742. doi:10. 1016/j.bbrc.2018.02.051

12. Luo D, Chen H, Li X, et al. Activation of the ROCK1/MMP-9 pathway is associated with the invasion and poor prognosis in papillary thyroid carcinoma. Int $J$ Oncol. 2017;51:1209-1218. doi:10.3892/ijo.2017.4100

13. Lacout A, Chevenet C, Marcy PY. Recurrence in patients with classic papillary thyroid carcinoma: highlight on power doppler US. Radiology. 2016;279:653-654. doi:10.1148/radiol.2016152506

14. Brunet VA, Pericay C, Moya I, et al. microRNA expression profile in stage III colorectal cancer: circulating miR-18a and miR-29a as promising biomarkers. Oncol Rep. 2013;30:320-326. doi:10.3892/ or.2013.2475

15. Li Y, Kong D, Ahmad A, et al. Epigenetic deregulation of miR-29a and miR-1256 by isoflavone contributes to the inhibition of prostate cancer cell growth and invasion. Epigenetics. 2012;7:940. doi:10.4161/epi.21236

16. Li R, Liu J, Li Q, et al. miR-29a suppresses growth and metastasis in papillary thyroid carcinoma by targeting AKT3. Tumor Biol. 2016;37:3987-3996. doi:10.1007/s13277-015-4165-9

17. Liu J, Fei D, Xing J, et al. MicroRNA-29a inhibits proliferation and induces apoptosis in rheumatoid arthritis fibroblast-like synoviocytes by repressing STAT3. Biomed Pharmacother;2017. 173-181. doi:10.1016/j.biopha.2017.09.120

18. Ma Y. Sun Y miR-29a-3p inhibits growth, proliferation, and invasion of papillary thyroid carcinoma by suppressing NF-kappaB signaling via direct targeting of OTUB2. Cancer Manag Res. 2019;11:13-23. doi:10.2147/CMAR.S184781

19. Ma J, Li T, Yuan H, et al. MicroRNA-29a inhibits proliferation and motility of Schwannoma cells by targeting CDK6. J Cell Biochem. 2017;119:2617-2626.
20. Mazyar J, Stanley Z, Chen WT. Plasma seprase and DPP4 levels as markers of disease and prognosis in cancer. Dis Markers. 2012;32:309-320. doi:10.3233/DMA-2011-0889

21. Drucker DJ. Dipeptidyl peptidase-4 inhibition and the treatment of type 2 diabetes preclinical biology and mechanisms of action. Diabetes Care. 2007;30:1335. doi:10.2337/dc07-0717

22. Tanaka Y, Kume S, Chin-Kanasaki M, et al. Renoprotective effect of DPP-4 inhibitors against free fatty acid-bound albumin-induced renal proximal tubular cell injury. Biochem Biophys Res Commun. 2016;470:539. doi:10.1016/j.bbrc.2016.01.109

23. Kanasaki K, Shi S, Kanasaki M, et al. Linagliptin-mediated DPP-4 inhibition ameliorates kidney fibrosis in streptozotocin-induced diabetic mice by inhibiting endothelial-to-mesenchymal transition in a therapeutic regimen. Diabetes. 2014;63:2120. doi:10.2337/db13-1029

24. Nakashima S, Matsui T, Takeuchi M, et al. Linagliptin blocks renal damage in type 1 diabetic rats by suppressing advanced glycation end products-receptor axis. Hormone Metab Res. 2014;46:717-721. doi:10.1055/s-0034-1371892

25. Ott C, Raff U, Schmidt S, et al. Effects of saxagliptin on early microvascular changes in patients with type 2 diabetes. Cardiovasc Diabetol. 2014;13:1-9. doi:10.1186/1475-2840-13-1

26. Mukai E, Morita A, Hiratsuka A, et al. Dual roles of a DPP-4 inhibitor on cytoprotection and proliferation of pancreatic beta cells in a mouse model of beta cell injury/regeneration. Diabetologia. 2014;57:S93-S93.

27. Wronkowitz N, Görgens SW, Romacho T, et al. Soluble DPP4 induces inflammation and proliferation of human smooth muscle cells via protease-activated receptor 2. Biochim Biophys Acta. 2014;1842:1613-1621. doi:10.1016/j.bbadis.2014.06.004

28. Davies S, Beckenkamp A. Buffon A CD26 a cancer stem cell marker and therapeutic target. Biomed Pharmacother. 2015;71:135-138. doi:10.1016/j.biopha.2015.02.031
OncoTargets and Therapy

\section{Publish your work in this journal}

OncoTargets and Therapy is an international, peer-reviewed, open access journal focusing on the pathological basis of all cancers, potential targets for therapy and treatment protocols employed to improve the management of cancer patients. The journal also focuses on the impact of management programs and new therapeutic

\section{Dovepress}

agents and protocols on patient perspectives such as quality of life, adherence and satisfaction. The manuscript management system is completely online and includes a very quick and fair peer-review system, which is all easy to use. Visit http://www.dovepress.com/ testimonials.php to read real quotes from published authors. 